\title{
DÉTECTION ET CARACTÉRISATION des tourbillons de sillage des avions par Lidar Doppler
}

Agnès DOLFI-BOUTEYRE Laurent LOMBARD Alexandre HALLERMEYER Matthieu VALLA Claudine BESSON

Département Optique Théorique et Appliquée (DOTA), ONERA agnes.dolfi-bouteyre@onera.fr

Dans un contexte où le trafic aérien s'accroît chaque jour, l'optimisation des débits en termes de décollage et d'atterrissage est devenue un enjeu majeur pour les aéroports à forte affluence. Ces débits sont principalement limités par la présence de tourbillons de sillage communément appelés wake vortex. Ceux-ci sont formés après le passage d'un avion et représentent un danger potentiel pour l'avion suivant. Afin d'éviter les accidents, les organisations de sûreté de la navigation aérienne ont défıni des distances de sécurité à respecter entre décollages ou atterrissages successifs. Ces distances prennent en compte la catégorie de poids des deux appareils en considérant des situations pirecas. De nombreuses études ont été menées pour permettre une meilleure compréhension de ces tourbillons et ont permis de constater que leur comportement varie en fonction des conditions atmosphériques, en particulier en fonction du vent et de la turbulence atmosphérique. Afin d'étudier la dynamique de la masse d'air et l'évolution de ces phénomènes, on utilise depuis une dizaine d'années le Lidar (light detection and ranging). Le Lidar pulsé est devenu l'instrument de référence pour la mesure à distance des tourbillons de sillage (positions et puissance).

l'ONERA travaille sur l'amélioration des performances de ces Lidars en termes de portée et de rapidité notamment en optimisant le traitement de signal et en développant des sources laser fibrées de nouvelle génération.

\section{Les tourbillons de sillages}

La Figure 1 est une illustration de ce que sont les tourbillons de sillage. Leur présence est ici visible du fait de l'ensemencement par des nuages. Ce phénomène physique résulte de la portance propre à tout objet volant possédant des ailes. Un avion porté par l'air laisse derrière lui un sillage constitué de deux tourbillons contra-rotatifs, d'autant plus puissants que la masse de l'appareil est importante.

Ces paires de tourbillons de sillage contra-rotatifs interagissent entre eux. Àl'intérieur de la paire, la composante

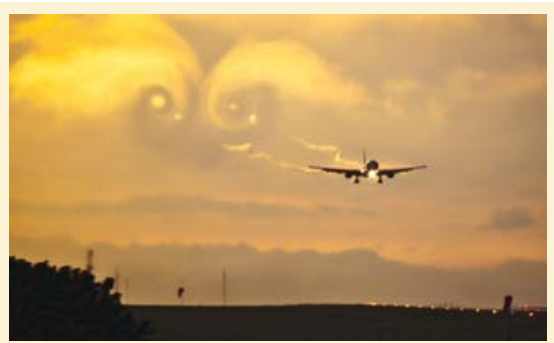

Figure 1. Tourbillons de sillage d'un Boeing 757 en phase d'atterrissage (source https://www.flickr.com/ photos/44073224@N04/). 


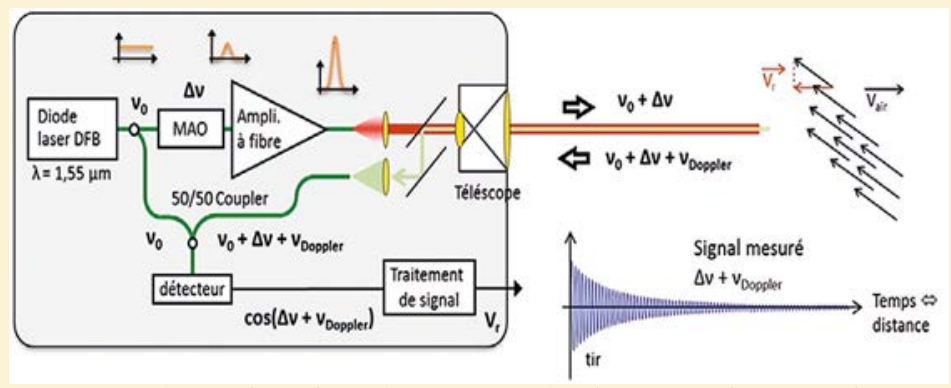

Figure 2. Schéma du Lidar cohérent Doppler de mesure de vitesse de vent résolu en distance.

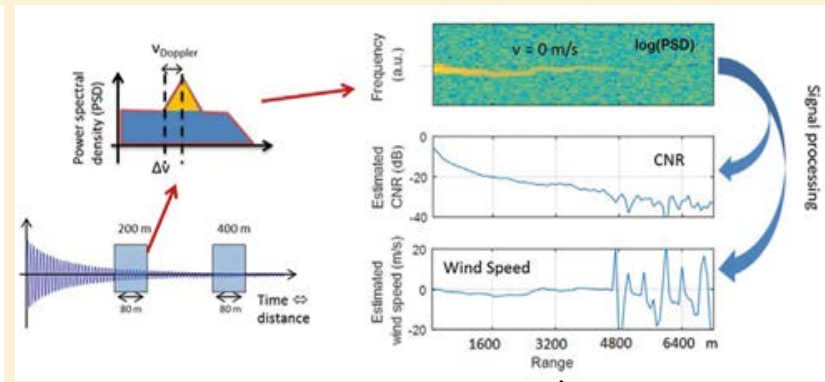

Figure 3. Principe du traitement de signal. À gauche DSP et signal temporel. À droite de haut en bas : périodogramme, CNR (carrier to noise ratio), vitesse moyenne par tranche de distance. verticale des vecteurs vitesse est orientée vers le bas. Le vecteur vitesse résultant est donc également dirigé vers le bas ce qui entraîne une descente de la paire de tourbillons.

La puissance contenue dans un tourbillon est exprimée par sa circulation notée $\Gamma\left(\mathrm{m}^{2} / \mathrm{s}\right)$. La circulation est l'intégrale curviligne du champ de vitesse sur un contour fermé (ici un cercle) :

$$
\Gamma=\oint_{C} \overrightarrow{\mathrm{V}} \cdot \overrightarrow{\mathrm{d} l}
$$

\section{Principe de \\ fonctionnement du Lidar Doppler cohérent pulisé}

Le Lidar cohérent pulsé est un outil précieux pour étudier ces tourbillons, et plus généralement pourétudier les mouvements d'air. Le Lidar, par définition, est la transposition dans le domaine optique du radar: une onde optique envoyée sur une cible (solide ou volumique) est partiellement rétrodiffusée, collectée puis analysée par l'instrument. Le lidar à détection directe mesure l'intensité du signal rétrodiffusé. Par exemple le lidar aérosols [2] mesure leur concentration, le lidar télémètre mesure un temps d'aller et retour (temps de vol) entre l' instrument et une cible solide, ce qui permet d'estimer une distance.

Les Lidars cohérents, en revanche, mesurent les interférences entre l'onde signal rétrodiffusée par la cible et une onde de référence prélevée sur le laser. L'analyse de ces interférences permet de mesurer une différence de fréquences due par exemple à un décalage Doppler. La figure 2 montre un montage typique de Lidar cohérent Doppler de mesure de vitesse de vent résolue en distance.
Une source laser continue de bonne qualité spatiale et spectrale émet le faisceau de référence à la fréquence $v_{0}$. Le faisceau est sculpté en impulsions de plusieurs centaines de nanosecondes grâce à un modulateur acousto-optique puis amplifié dans un amplificateur fibré. Avec les amplificateurs de nouvelle génération, des puissances crêtes de l'ordre du $\mathrm{kW}$ sont émises. Un télescope permet d'émettre et de recevoir le faisceau rétrodiffusé sur les particules en suspension dans l'air. La lumière collectée est mélangée avec le faisceau de référence prélevéen sortie de laser (l'oscillateur local « OL »). Un détecteur mesure le battement temporel entre ces deux faisceaux.

La lumière collectée est décalée en fréquence d'une part de $\Delta v$ par le modulateur acousto-optique (typiquement quelques dizaines de $\mathrm{MHz}$ ) et d'autre part par l'effet Doppler des particules sur le faisceau laser:

$$
\begin{gathered}
v_{\text {Doppler }}=-2 V_{r} / \lambda=-V_{r} \cdot 1,3 \mathrm{MHz} /(\mathrm{m} / \mathrm{s}) \\
\text { à } 1,55 \mu \mathrm{m}
\end{gathered}
$$

où $V r$ est la vitesse des particules (supposée être égale à la vitesse du vent) projetée sur l'axe optique. $v_{\text {Doppler }}$ est positif quand les particules se dirigent vers l'instrument et négatif dans le cas contraire. Le signal vu par le détecteur

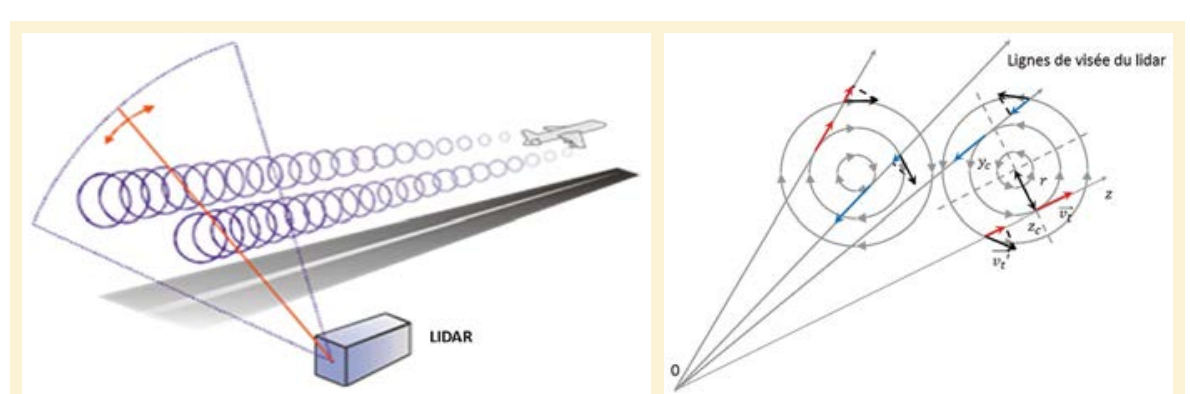

Figure 4. (a) Configuration du plan de mesure Lidar. (b) Projections des vitesses sur l'axe de mesure. est un battement à la fréquence différence, c'est-à-dire à $\Delta v+v_{\text {Doppler }}$ dont l'amplitude décroît avec la distance. Le signal temporel est schématisé dans l'encart de la figure. En réalité, le bruit est bien supérieur au signal et seul un traitement adapté permet de remonter à la vitesse $V_{r}$ en fonction de la distance. Le traitement est illustré à la figure 3.

Le traitement consiste à découper des tranches temporelles correspondant à des tranches de distance, par exemple ici autour de $200 \mathrm{~m}$. La densité spectrale de puissance (DSP) du signal de chaque bin est calculée et sommée sur des milliers de tirs. Le périodogramme est une simple concaténation des DSP mises en colonne pour chaque tranche (en abscisse).

Un ou plusieurs estimateurs sont alors mis en œuvre pour identifier la position et l'énergie contenue dans le pic signal. Les résultats sont respectivement la fréquence centrale, donc la vitesse du vent et le CNR (carrier to noise ratio) qui permet d'estimer la qualité de l'information de vitesse. Un exemple de résultat de ces estimations est montré à la figure 3 en bas à droite : au-delà de $5000 \mathrm{~m}$, le CNR est trop faible et la vitesse de vent comprend de nombreux points aberrants qui indiquent 


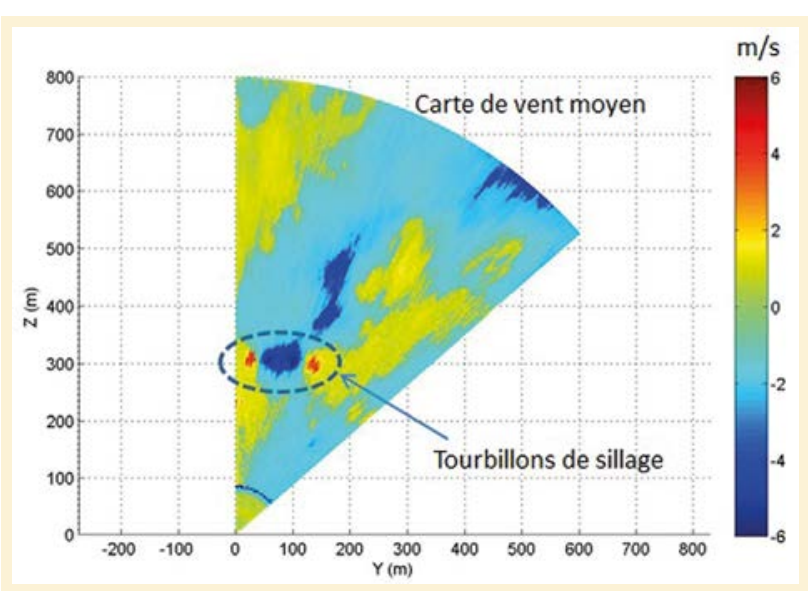

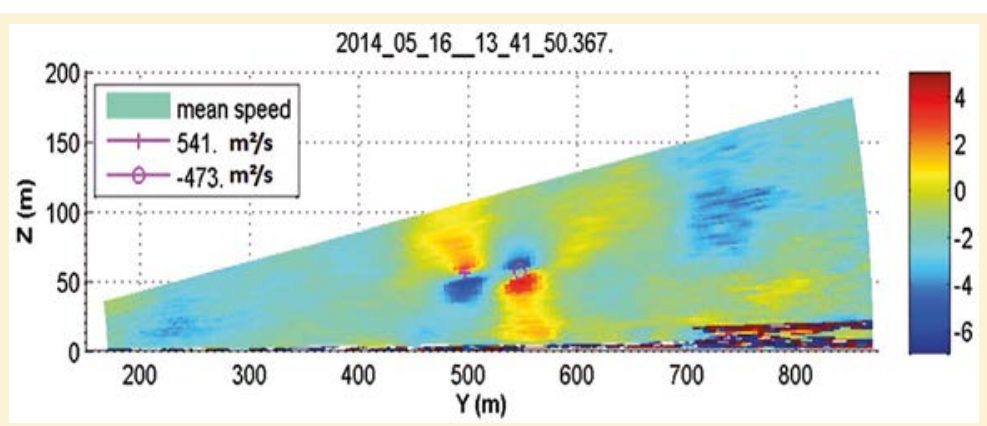

Figure 5. Carte de champ de vent dans le plan vertical Y0Z mesuré par Lidar à l'aéroport Charles de Gaulle. Tourbillon localisé à $300 \mathrm{~m}$ d'altitude environ.

Figure 6. Carte de champ de vent dans le plan vertical Y0Z mesuré par Lidar à l'aéroport Toulouse Blagnac. Tourbillon localisé à $40 \mathrm{~m}$ d'altitude environ. la portée du Lidar. Cette portée dépend de la puissance et de la qualité de la source laser mais aussi des conditions météorologiques. Typiquement, un jour clair et peu pollué n'est pas favorable pour la portée du Lidar. En revanche, une brume légère est favorable.

Les records de portée des Lidars vent obtenus à l'ONERA par temps favorable sont des profils de vitesse allant jusqu'à $15 \mathrm{~km}$, obtenus en tir horizontal en 100 ms grâce aux sources laser de nouvelle génération.

\section{Détection des touribillons parr Lidar}

Le Lidar Doppler mesure la projection sur l'axe laser de la vitesse de la masse d'air. Il est en général situé sous la trajectoire des avions ou sur le côté de la piste. La détection est effectuée sur un plan unique orthogonal à la trajectoire de l'avion (Figure 4a).

La géométrie de cette configuration de détection transverse est illustrée en Figure $4 b$ avec un Lidar positionné en 0 . Les vitesses moyennes sont positives à l'extérieur des deux centres des vortex (flèches rouges : la masse d'air s'éloigne du Lidar) et négatives entre les deux centres (flèches bleues). La Figure 5 montre un champ de vent mesuré par Lidar, où l'on voit la présence d'une paire de tourbillons de sillage d'un avion en phase d'approche à une altitude de $300 \mathrm{~m}$ environ. La paire de vortex se reconnaît par le lobe bleu (vitesses négatives) entouré par deux lobes rouges (vitesses positives) comme illustré géométriquement à la figure $4 b$.
La Figure 6 montre une mesure de tourbillon de sillage pour un avion proche de l'atterrissage à une altitude de 30 ou $40 \mathrm{~m}$. Le Lidar est situé avant le seuil de piste et latéralement à environ $550 \mathrm{~m}$ de l'axe de la piste.

Ces mesures ont été effectuées par un Lidar Windcube de Leosphere lors d'une campagne de mesures dans le cadre du projet de recherche Européen SESAR (Single European Sky ATM Research). Un traitement du signal spécifique a été implémenté par l'ONERA afin d'estimer de façon précise les circulations et les positions des tourbillons [3-5]. Les positions sont indiquées par une croix et un rond rose à la figure 6 et les valeurs des circulations en $\mathrm{m}^{2} / \mathrm{s}$ sont données dans l'encadré. Ce traitement est basé sur l'analyse détaillée des spectres de fréquence. Un traitement dit « paramétrique » est basé sur un simulateur de réponse Lidar et un modèle de tourbillon de sillage dont on fait varier les paramètres (positions et circulations) jusqu'à ce que les spectres obtenus correspondent à ceux mesurés. La précision en position est de l'ordre du mètre, bien que la résolution spatiale intrinsèque du Lidar donnée par la durée des impulsions soit de 50 à 100 $\mathrm{m}$. L'erreur relative sur l'estimation de la circulation, lorsque le vortex n'est pas encore déformé par la turbulence atmosphérique, est de l'ordre de $10 \%$.

\section{Conclusion}

Le Lidar cohérent pulsé est un instrument bien adapté à la caractérisation des tourbillons de sillages des aéronefs sur site aéroportuaire. Des mesures de tourbillons avec un Lidar embarqué sur l'avion en configuration transverse ou axiale sont également possibles [6]. Les prochains défis pour des Lidars fibrés sur les aéroports sont la cartographie rapide de vents $\left(360^{\circ}\right.$ en $\left.1 \mathrm{mn}\right)$ à longue distance $(>10 \mathrm{~km})$ autour des sites aéroportuaires, et la cartographie de la turbulence de vent afin de pourvoir anticiper les trajectoires et prévoir les durées de vie des tourbillons de sillage.

\section{RÉFÉRENCES}

[1] S.M. Hannon, J.A. Thomson, Aircraft wake vortex detection and measurement with pulsed solid-state coherent laser radar, J. Mod. Opt. 41, pp. 2175-2196, 1994

[2] J.P. Cariou, L. Thobois, P. Royer, Détection et analyse automatique des aérosols atmosphériques par Lidar ultraviolet et infraronge, Photoniques 82, juin/août 2016

[3] S. Rahm, I. Smaliko, F. Kopp, Characterization of aircraft wake-vortices by airborne coherent Doppler Lidar, J. Aircraft 44, pp. 799-805, 2007

[4] R. Frehlich, R. Sharman. Maximum likelihood estimates of vortex parameters from simulated coherent doppler lidar data. Journal of Atmospheric and Oceanic Technology 22, p. 117130, 2005

[5] A. Hallermeyer et al., Development and assessment of a Wake Vortex characterization algorithm based on a bybrid Lidar signal processing, ${ }^{\text {th }}$ AIAA Atmospheric and Space Environments Conference, 2016

[6] A. Dolfi-Bouteyre et al., Pulsed 1.5- $\mu m$ Lidar for axial aircraft WakeVortex detection based on high-brightness large-core fiber amplifier, IEEE Journal of Selected Topics in Quantum Electronics 15, march/april 2009 